Original article

\title{
Gut microbiome and magnetic resonance spectroscopy study of subjects at ultra-high risk for psychosis may support the membrane hypothesis
}

\author{
Ying He ${ }^{\mathrm{a}, \mathrm{b}}$, Tomasz Kosciolek ${ }^{\mathrm{c}}$, Jinsong Tang ${ }^{\mathrm{a}}$, Yao Zhou ${ }^{\mathrm{a}}$, Zongchang $\mathrm{Li}^{\mathrm{a}}$, Xiaoqian Ma ${ }^{\mathrm{a}}$, \\ Qiyun Zhu' ${ }^{\mathrm{c}}$, Ning Yuan ${ }^{\mathrm{a}}$, Liu Yuan ${ }^{\mathrm{a}}$, Chunwang $\mathrm{Li}^{\mathrm{d}}$, Ke Jin ${ }^{\mathrm{d}}$, Rob Knight ${ }^{\mathrm{c}}$, \\ Ming T. Tsuang ${ }^{\mathrm{b}, \mathrm{e}}$, Xiaogang Chen ${ }^{\mathrm{a}, \mathrm{f}, \mathrm{g}, \mathrm{h}, *}$ \\ a Department of Psychiatry, the Second Xiangya Hospital, Central South University, Changsha, Hunan 410011, China \\ ${ }^{\mathrm{b}}$ Center for Behavioral Genomics, Department of Psychiatry, Institute for Genomic Medicine, University of California San Diego, La Jolla, CA, USA \\ ${ }^{\mathrm{c}}$ Department of Pediatrics, University of California San Diego, San Diego, CA 92110, USA \\ d Department of Radiology, Hunan Childen's Hospital, Changsha 410007, China \\ e Harvard Institute of Psychiatric Epidemiology and Genetics, Harvard School of Public Health, Boston, MA, USA \\ ${ }^{\mathrm{f}}$ Mental Health Institute of Central South University, Changsha, Hunan 410011, China \\ g China National Clinical Research Center on Mental Disorders (Xiangya), Changsha, Hunan 410011, China \\ ${ }^{\text {h }}$ China National Technology Institute on Mental Disorders, Changsha, Hunan 410011, China
}

\section{A R T I C L E IN F O}

\section{Article history:}

Received 28 February 2018

Received in revised form 7 May 2018

Accepted 25 May 2018

Available online 2 June 2018

\section{Keywords:}

Schizophrenia

Ultra-high risk

Microbiome

Magnetic resonance spectroscopy

\begin{abstract}
A B S T R A C T
Background: The microbiota-gut-brain axis and membrane dysfunction in the brain has attracted increasing attention in the field of psychiatric research. However, the possible interactive role of gut microbiota and brain function in the prodromal stage of schizophrenia has not been studied yet.

Methods: To explore this, we collected fecal samples and performed Magnetic Resonance Spectroscopy (MRS) scans in 81 high risk (HR) subjects, 19 ultra-high risk (UHR) subjects and 69 health controls (HC). Then we analyzed the differences in gut microbiota and choline concentrations in the anterior cingulate cortex (ACC).

Results: Presences of the orders Clostridiales,Lactobacillales and Bacteroidales were observed at increase levels in fecal samples of UHR subjects compared to the other two groups. The composition changes of gut microbiota indicate the increased production of Short Chain Fatty Acids (SCFAs), which could activate microglia and then disrupt membrane metabolism. Furthermore, this was confirmed by an increase of choline levels, a brain imaging marker of membrane dysfunction, which is also significantly elevated in UHR subjects compared to the HR and HC groups.

Conclusion: Both gut microbiome and imaging studies of UHR subjects suggest the membrane dysfunction in the brain and hence might support the membrane hypothesis of schizophrenia.
\end{abstract}

(c) 2018 Elsevier Masson SAS. All rights reserved.

\section{Introduction}

Schizophrenia is a serious mental disorder that usually develops in late adolescence or early adulthood. Characterized by delusions, hallucinations and cognitive impairments, schizophrenia affects nearly $1 \%$ of the world's population [1]. In genetic analysis, monozygotic twins studies only show around $50 \%$ concordance rate which strongly suggests that genetic basis alone is insufficient in explaining the development of schizophrenia [2]. Therefore, this

\footnotetext{
* Corresponding author at: Department of Psychiatry, the Second Xiangya Hospital, Central South University, Changsha, Hunan 410011, China.

E-mail address: Chenxiaogang@csu.edu.cn (X. Chen).
}

prompts the investigation of environmental risk factors. Notable environmental factors include perinatal complications, neurotrauma, psycho-trauma, substance abuse, and migration among others. However, one of the most important and immediate environmental factor is the gut microbiota [3]. The human gut microbiome comprises of bacteria on the order of trillions in magnitude with between 3 and 10 million unique genes, dwarfing the human genome by a ratio of 150:1 [4-7]. This massive genetic repertoire which, unlike the human genome, is influenced by medication, probiotic or prebiotic supplementation or lifestyle changes, provides a means to better understand a variety of diseases and also to create low-cost rapid therapies with minimal side-effects.

The concept of the microbiota-gut-brain axis was proposed nearly a decade ago [8]. Considerable evidence now supports that 
there is a bidirectional interaction between the nervous system and the enteric microbiota. Four main routes have been suggested: neural, endocrine, immune, and metabolic pathways [9,10].

Besides the relatively new microbiota-gut-brain axis concept, the origin of schizophrenia has many classic hypotheses, such as the dopamine (DA) hypothesis [11], serotonin (5-HT) hypothesis [12], glutamate hypothesis [13] and membrane hypothesis [14]. Thus, what are the relationships between microbiota-gut-brain axis and hypotheses of schizophrenia? The microbiota-gut-brain axis could be related to the classic neurotransmitter hypotheses due to the fact that microbes produce essential neurotransmitters such as DA, 5-HT and norepinephrine [15]. In fact, more than $90 \%$ of human $5-\mathrm{HT}$ is produced in the gut where the microbiome promotes its synthesis and regulation $[16,17]$. In contrast, the role of the microbiota-gutbrain axis in the membrane hypothesis of schizophrenia is still unknown. The membrane hypothesis of schizophrenia is not only the most inclusive and promising hypothesis but also the biochemical basis of neurodevelopmental disorders. The core of the membrane hypothesis is that reduced synthesis and increased degradation of cell membranes, and the subsequent abnormal signal transmission are the causes of schizophrenia [14,18]. Furthermore, the membrane hypothesis also suggests that the schizophrenia is part of a systemic disease of membrane dysfunction instead of an independent brain disease, which happens to fit the key point of microbiota-gut-brain axis [14]. However, unlike the classic neurotransmitter hypotheses, the relationship with microbiota-gut-brain axis and membrane hypothesis is still lack of research. In order to fill this research gap, we simultaneously measured the composition of gut microbiota and the marker of cell membrane disruption in brain, which is choline [19]. As Schwarz et.al and Shen et.al have already conducted the gut microbiome study of first episode psychosis and schizophrenia $[20,21]$, we proposed to study the gut microbiota and brain imaging features of schizophrenia-afflicted individuals by following the highrisk (HR) and ultra-high risk (UHR) subjects, thereby exploring the relationships of microbiota-gut-brain axis and membrane hypothesis as well as shedding light on a new perspective for schizophrenia research. Here, we report the baseline results of our longitudinal prospective observational study.

\section{Methods}

\subsection{Participants}

Eighty-one HR subjects, nineteen UHR subjects and sixty-nine health controls (HC) were recruited from November 2016 to May 2017 at the Second Xiangya Hospital, Central South University,
Changsha, Hunan, China. All participants were $13-30$ years old Han Chinese; the demographic characteristics are detailed in Table 1.

All HCs and HRs were screened for lifetime absence of all fiveaxis diagnoses of mental disorders using the DSM-IV-TR criteria [22]. In addition, all HCs were confirmed to have no family history of psychiatric disorder in their first-degree relatives. By contrast, the HRs were confirmed to have at least one of their first-degree relatives diagnosed with schizophrenia. All UHR subjects were screened by the Structured Interview for Prodromal Syndromes (SIPS) [23] and fulfilled one of the three subsets: Brief Intermittent Psychotic Syndrome (BIPS), Attenuated Positive Symptom Syndrome (APSS) and Genetic Risk and Deterioration Syndrome (GRDS). Exclusion criteria for all subjects included: gastrointestinal and endocrine diseases (including constipation and diarrhea), serious organ disorders (such as stroke and heart failure), a history of diagnosis of psychiatric disorders and corresponding treatments (such as antipsychotics, antidepressants and anticonvulsants), had used alcohol, antibiotics, probiotics or any other medications (oral or injectable) during the last three months.

This study was approved by the Ethics Committee of the Second Xiangya Hospital, Central South University (No. S090, 2016) and carried out in accordance with the Declaration of Helsinki. All participants were aware of the risks and benefits of the study and signed informed consent forms. Thirty-seven HCs did not agree with the Proton Magnetic Resonance Spectroscopy $\left({ }^{1} \mathrm{H}-\mathrm{MRS}\right)$ scanning, but they all had completed the clinical assessment and fecal sample collection. And there were no statistical differences of characteristics between the HCs which agreed to brain scanning and those who did not (see Table S1 in Supplementary material).

\subsection{Clinical assessment}

Both HRs and UHRs completed the Scale of Prodromal Symptoms (SOPS) for screening of schizophrenic symptoms and were assessed with the Global Assessment of Function Scale, Modified Version (GAF-M) to evaluate their psychological, social, and occupational functionalities [24]. The SOPS is a 19-item scale, a part of the SIPS and includes four subscales for positive, negative, disorganized and general symptoms.

\subsection{Fecal DNA extraction and sequencing}

All samples were collected using Swube ${ }^{\mathrm{TM}}$ dual swabs and stored at $-80^{\circ} \mathrm{C}$ for further processing. Each $0.2 \mathrm{~g}$ of raw sample was used for DNA extraction with QIAGEN QIAamp kit. We evaluated DNA concentration in each sample by fluorometry and

Table 1

General characteristics of the recruited subjects.

\begin{tabular}{|c|c|c|c|c|c|}
\hline Variable & $\mathrm{HR}(\mathrm{n}=81)$ & $\operatorname{UHR}(\mathrm{n}=19)$ & $\mathrm{HC}(\mathrm{n}=69)$ & Statistic value (ChiSq or W) & $p$-value \\
\hline $\begin{array}{l}\text { Age, years } \\
\qquad(\text { mean } \pm \text { S.D. })\end{array}$ & $21.67 \pm 5.75$ & $20.47 \pm 4.57$ & $23.13 \pm 3.89$ & 4.56 & 0.103 \\
\hline $\begin{array}{l}\text { Gender } \\
\quad \text { (male, female) }\end{array}$ & $41 \mathrm{M}, 40 \mathrm{~F}$ & $15 \mathrm{M}, 4 \mathrm{~F}$ & $37 \mathrm{M}, 32 \mathrm{~F}$ & 5.08 & 0.079 \\
\hline $\begin{array}{l}\text { P score } \\
\quad(\text { mean } \pm \text { S.D. })\end{array}$ & $0.81 \pm 1.48$ & $11.47 \pm 6.76$ & - & 24.5 & $<0.001$ \\
\hline $\begin{array}{l}\text { N score } \\
\quad(\text { mean } \pm \text { S.D. })\end{array}$ & $1.8 \pm 3.79$ & $10.26 \pm 5.13$ & - & 120 & $<0.001$ \\
\hline $\begin{array}{l}\text { D score } \\
\quad(\text { mean } \pm \text { S.D. })\end{array}$ & $0.63 \pm 1.23$ & $4.89 \pm 4.48$ & - & 186 & $<0.001$ \\
\hline $\begin{array}{l}\text { G score } \\
\quad(\text { mean } \pm \text { S.D. })\end{array}$ & $0.95 \pm 1.35$ & $4.37 \pm 3.52$ & - & 300.5 & $<0.001$ \\
\hline $\begin{array}{l}\text { GAF score } \\
\qquad(\text { mean } \pm \text { S.D. })\end{array}$ & $84.17 \pm 6.45$ & $58.89 \pm 11.73$ & - & 1453.5 & $<0.001$ \\
\hline
\end{tabular}

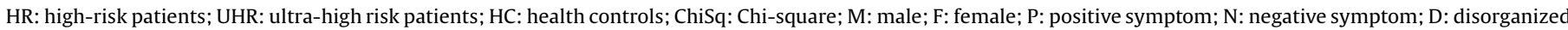
symptom; G: general symptom; GAF: Global Assessment of Function Scale.

The difference between the groups is significant with a $p$-value less than 0.01 . 
sample integrity by agarose gel electrophoresis ( $1 \%$ concentration, $150 \mathrm{~V}$, for $40 \mathrm{~min}$ ). PCR amplification and library preparation were performed using 515F/806R primers to target the V4 region of the $16 S$ rRNA gene. Samples were amplified in triplicate and cleaned using AGENCOURT AmpureXPbeads kit. For each reaction, we used $30 \mathrm{ng}$ of DNA, $4 \mu \mathrm{L}$ of PCR primer cocktail and $25 \mu \mathrm{L}$ of NEB Phusion High-Fidelity PCR master mix. The final library was quantified by determining the average molecule length using Agilent 2100 bioanalyzer and quantified by real-time EvaGreen qPCR. Sequencing was performed using Illumina MiSeq 250 base-pair paired-end sequencing by BGI Genomics, China.

\section{4. ${ }^{1} \mathrm{H}$ MRS scanning procedure}

All participants had Proton Magnetic Resonance Spectroscopy $\left({ }^{1} \mathrm{H}-\mathrm{MRS}\right)$ scans within $24 \mathrm{~h}$ after attending the study. All examinations were conducted on a 3.0 T magnetic resonance imager (Siemens, Skyra, Germany) using a 16-channel head coil at the Magnetic Imaging Centre of Hunan Children's Hospital. Participants were equipped with foam pads to reduce head motion and scanner noise. Anatomical images were acquired with threedimensional T1-weighted magnetization-prepared rapid acquisition gradient echo for voxel tissue segmentation (TR $2530 \mathrm{~ms}$; TE $2.33 \mathrm{~ms}$; FOV $256 \times 256 \mathrm{~mm}$; flip angle $7^{\circ}$; slice thickness $1 \mathrm{~mm}$; gap $0 \mathrm{~mm}$; NEX 1 ; slices 192). ${ }^{1} \mathrm{H}$ MRS spectra were measured by using the standard PRESS sequence (svs_se, TR $3000 \mathrm{~ms}$, TE $30 \mathrm{~ms}$, NEX 80 ). A $10 \times 20 \times 20 \mathrm{~mm}$ voxel of interest (VOI) was located on gray matter of the anterior cingulate cortex (ACC) using coronal, sagittal and transverse images (Fig. 1). The unsuppressed water spectra were acquired from the same voxel.

Choline was quantified by using LCModel version $6.3-1 \mathrm{~B}$ (LCMODEL Inc. CA) at the Second Affiliated Hospital, Shantou University Medical College located in Shantou, Guangdong, China. The absolute choline concentration was calculated with tissue water as the internal reference and corrected for the cerebrospinal fluid (CSF) content in ACC. According to LCModel manual (http://s-provencher.com/pages/lcm-manual.shtml), seven HRs and two UHRs' spectra were discarded because of these exclusion criteria: (1) the Cramer-Rao minimum variance $>15 \%$, (2) choline FWHM $<0.1$, and (3) the signal-to-noise ratio $(\mathrm{SNR})>10$.

\subsection{Bioinformatics and statistical analysis}

To conduct 16S rRNA analysis, we first used QIIME2 to generate the feature table (BIOM), using the DADA2 sub-OTU (Operational Taxonomic Unit) picking an algorithm with default parameters $[25,26]$. On the output BIOM table, firstly, we calculated alpha- and beta-diversity metrics using QIIME2. Alpha-diversity is the species diversity within a single sample, here we used the observed OTUs to represent the richness and Shannon diversity index to quantify both abundance and evenness of the species. Beta-diversity is the differences in species composition among samples and the comparisons usually be presented in a distance matrix. Canberra distance matrix was used in the Principal Coordinates Analysis (PCoA) plot and the subsequent permutational analysis of variance (PERMANOVA) model was employed for testing the difference between groups. Then we applied Partial Least Squares Discriminant Analysis (PLS-DA) with mixOmics (https://CRAN.R-project. org/package=mixOmics) to divide the samples into different predefined clusters and employed sparse PLS-DA (sPLS-DA) to select the most discriminative features that help cluster samples. Linear Discriminant Analysis (LDA) Effect Size (LEfSe) [27] (http:// huttenhower.sph.harvard.edu/lefse) was performed to detect which taxa had significantly different abundances among groups with non-parametric factorial Kruskal-Wallis (KW) rank-sum test. For LEfSe analysis, we performed the LDA to estimate the effect size of each differentially abundant microbes with default settings (the non-negative threshold for the logarithmic LDA score was 2 and tests of significance were two-sided, $p$-values were less than 0.05 ). The effect size of LDA model is equivalent to the microbes's variability and discriminatory power between groups [25].

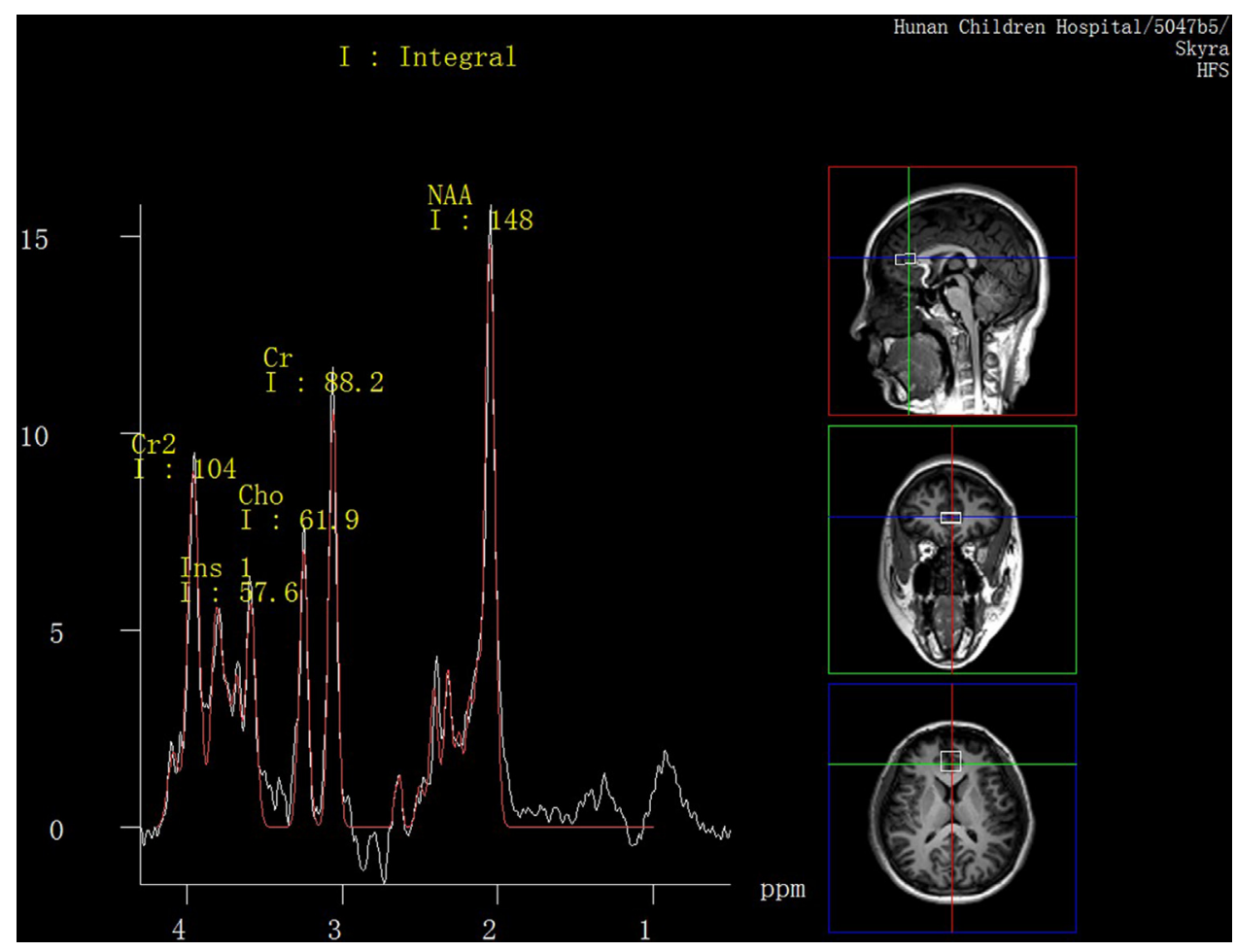

Fig. 1. MR images of the location of anterior cingulate cortex (ACC) voxel and spectral line of metabolites. 
PICRUSt was used to predict functional profiling of microbiota from 16S rRNA data with the Greengenes database (http://greengenes. lbl.gov) and KEGG Database (www.genome.jp/kegg/) [28]. And statistical analysis of PICRUSt outputs was performed with the Statistical Analysis of Metagenomic Profiles (STAMP) software package [29].

All subsequent statistical analyses were completed using the software package $\mathrm{R}$ (http://cran.r-project.org/). Demographic characteristics and clinical assessment scores were described and detected the statistical differences using the Chi-square test, the Kruskal-Wallis rank-sum test and the Wilcoxon rank-sum test. Differences in choline concentration in ACC were assessed with Analysis of Variance (ANOVA) and Analysis of Covariance (ANCOVA), and applied with post-hoc Tukey Honestly Significant Difference (HSD) test to correct for multiple comparisons.

\section{Results}

\subsection{Clinical characteristics}

The 3 groups (HR, UHR and HC) recruited in this study had no significant differences in age and gender $(p>0.05)$. However, significantly more severe symptoms and lower function were observed in UHR group (Table 1).

\subsection{Bioinformatics analysis of the sequences}

From a total of 169 samples, we obtained 6,514,098 qualified sequences at a minimum depth of 31,121 reads. Because of the high number of minimum reads, we selected it as a rarefaction depth and performed all subsequent analyses using a rarefied feature table.
First, we calculated global diversity metrics to quantify microbial differences between subject groups. For alpha-diversity, or within-subject diversity, we employed the observed OTUs and Shannon diversity index to estimate the microbial richness and diversity, respectively. We observed no statistically significant differences among the three groups in microbial richness (KruskalWallis $p=0.61$ ), or diversity (Kruskal-Wallis $p=0.97$ ). For betadiversity, we proceeded with the PCoA using Canberra distance matrix [30] and found that group difference was significant through PERMANOVA ( $\mathrm{p}=0.029$ ), in which, both the UHRs and HRs had significant differences with HCs ( $p=0.048$ for both).

Next, we applied PLS-DA to divide the samples into different clusters (Fig. 2, A) and used sPLS-DA to identify the most significantly abundant microbes in the UHR group (Fig. 2, B). The cluster plot showed heterogeneity within UHR group, which differed in distances from the other two groups. Combined with the LEfSe biomarker identification (Fig. 3), we found that orders Clostridiales, Lactobacillales and Bacteroidales and genera Lactobacillus and Prevotella were increased in UHRs compared to HRs and HCs. At the species level, only Lactobacillus ruminis was identified by both methods together as a significant feature in the UHR group.

Finally, we used PICRUSt to make an overall functional prediction at 3 levels into KEGG pathways (see http://www.genome.jp/kegg/ pathway.html\#mapping for the detailed pathways in all levels). However, none of the 3 levels showed any significant differences of functional coverage among three groups. Then the KEGG Orthology (KO) database (http://www.genome.jp/kegg/ko.html) was applied to address the molecular-level functions, such as the synthesis of DA and 5-HT as well as the metabolism of choline. As the synthesis of SCFAs are much more complicated than the others, partly because the SCFAs is a group of metabolites including acetate, propionate,
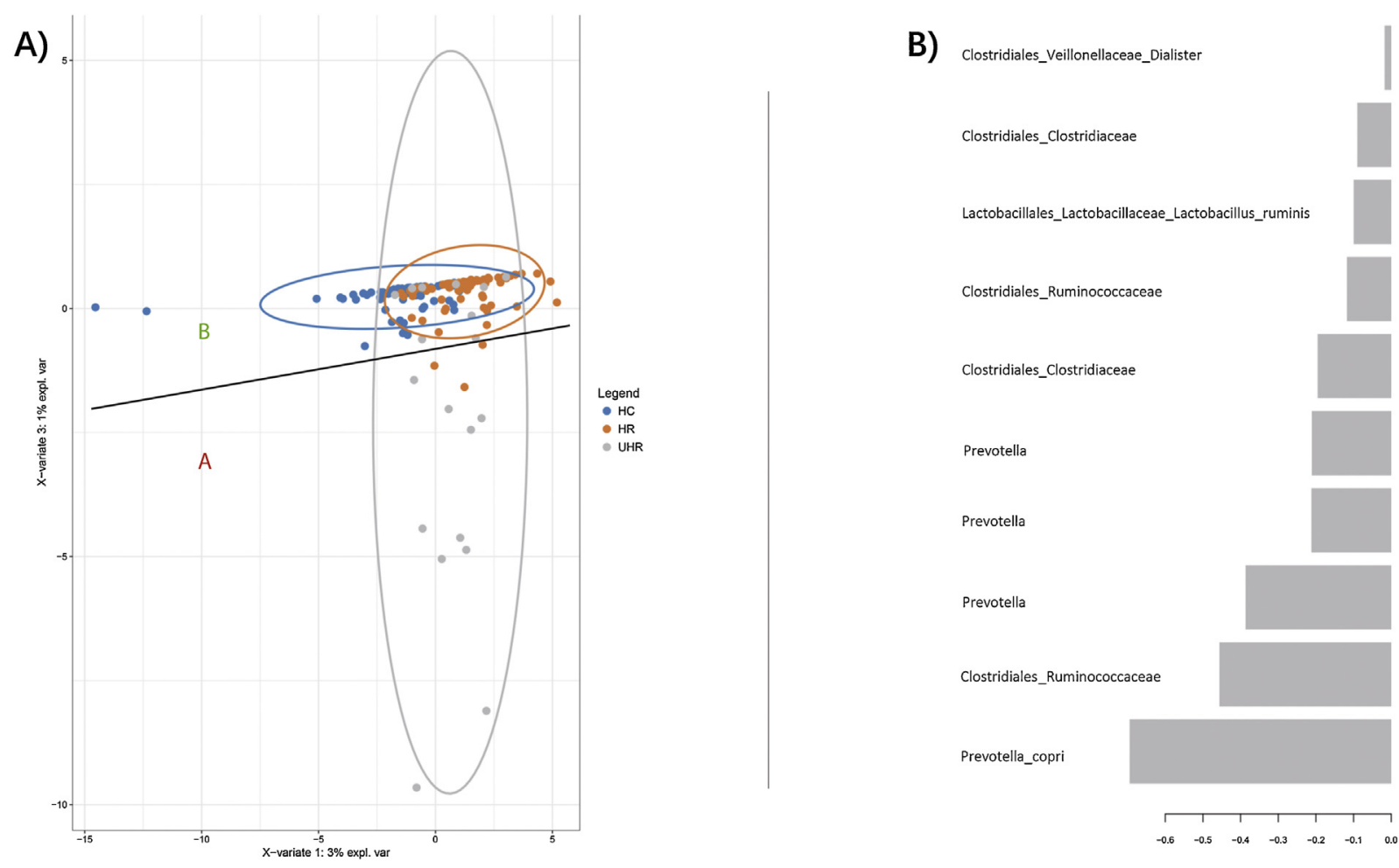

Fig. 2. Taxonomic differences between UHR, HR and HC groups with PLS-DA and sPLS-DA.

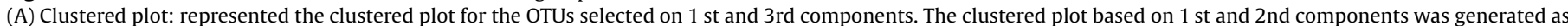

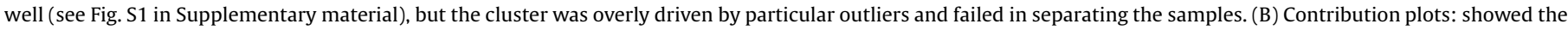

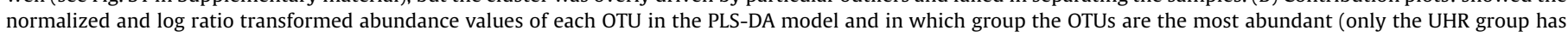
significant abundant OTUs in our model).

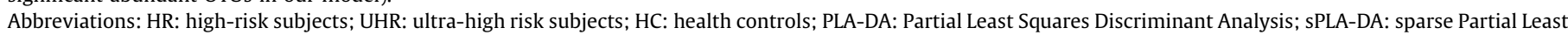
Squares Discriminant Analysis; OTU: Operational Taxonomic Unit. 
A)

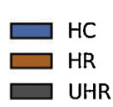

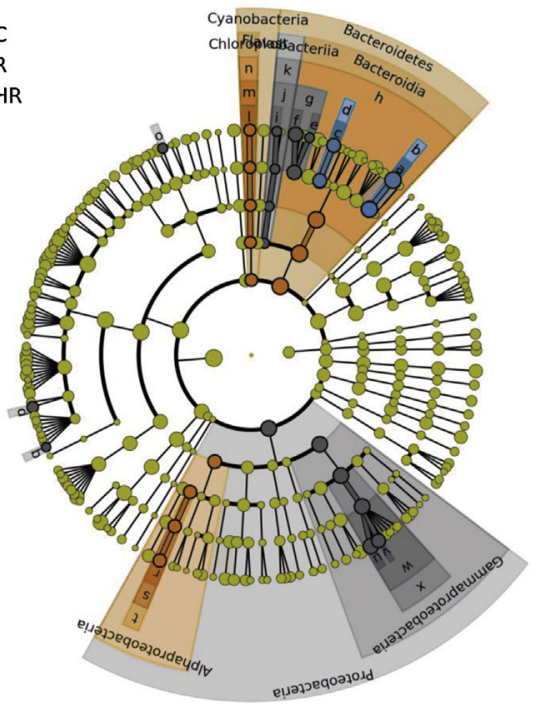

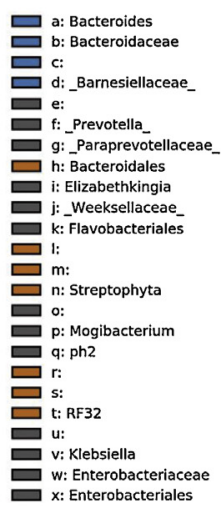

B)

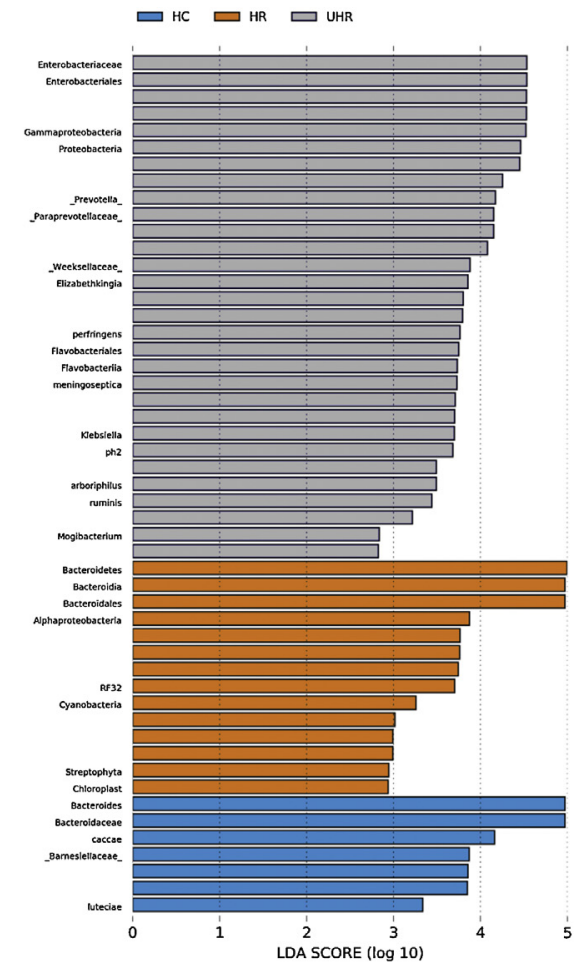

Fig. 3. Taxonomic differences between UHR, HR and HC groups with LEfSe.

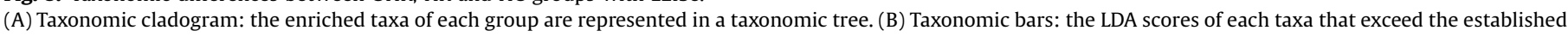
significance threshold.

Abbreviations: HR: high-risk subjects; UHR: ultra-high risk subjects; HC: healthy controls; LEfSe: Linear Discriminant Analysis (LDA) Effect Size.

butyrate and so on, so the simplified pathways of SCFAs production are adopted from Stilling et.al and Macfarlane et. al's papers instead of KO database [31,32]. And it turned out that instead of the synthesis of DA and 5-HT and the metabolism of choline, but the synthesis of SCFAs presented a difference among groups. That is, the SCFAs related pyruvate synthesis, acetyl-CoA synthesis and fatty acid biosynthesis initiation pathways were found to be elevated in UHR subjects other than HRs and HCs. But only the acetyl-CoA synthesis pathway showed a significant difference $(p=0.05)$ after multiple testing adjustment (Storey FDR; see Fig. S2 in Supplementary material).

\section{3. ${ }^{1} \mathrm{H}$ MRS spectra: comparison of choline levels between groups}

After quality control, there were 74 HR subjects, 17 UHR subjects and $32 \mathrm{HCs}$ left in the MRS cohort. With age and a dummy variable of gender serving as covariates, there was a significant difference of choline levels between three groups $(p=0.03)$. And after post-hoc analysis, we found the differences mostly derived from UHR and HC groups where the choline levels in UHR subjects were significantly higher than that of the HCs $(p=0.03)$ (Fig. 4).

\subsection{Association with gut microbiome, clinical symptoms and choline} concentration in anterior cingulate cortex

As illustrated in Fig. 2, the microbiota composition among groups was heterogeneous, but the underlying reason was unknown. To determine whether this was related with clinical symptoms or choline concentrations we identified 13 individuals (10 UHRs and 3 HRs) that clustered outside the main distribution area and 87 individuals ( 9 UHRs and 78 HRs) that gathered inside (Fig. 2). We found that the outside cluster does have significantly higher positive $(p<0.01)$, negative $(p<0.01)$ and dissociative $(p<0.01)$ symptom scores and lower GAF scores $(p<0.01)$. However, the general symptom scores $(p=0.15)$ and the choline concentrations $(p=0.53)$ were non-significantly increased in the outside cluster.

\section{Discussion}

\subsection{Relationships with previous studies}

As the first gut microbiome study of UHR subjects for schizophrenia, our results are consistent with previous gut microbiome studies of first episode psychosis, schizophrenia

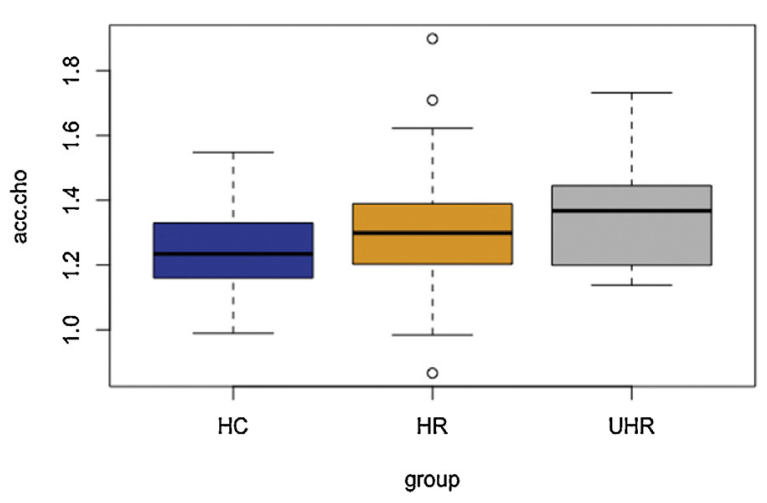

Fig. 4. The difference of choline levels in ACC between groups. Choline levels are significantly higher in UHR group than HC group.

Abbreviations: HR: high-risk subjects; UHR: ultra-high risk subjects; HC: health controls; ACC: anterior cingulate cortex; Cho: choline. 
and autism which reported elevated Lactobacillus [20,21,33-35], Clostridium [36-39], and Prevotella [20] levels in patients. Also, two oropharyngeal microbiome studies in schizophrenia revealed that Lactobacilli and Lactobacillus phage phi adh are more abundant in patients than controls $[40,41]$. The murine model of autism also showed that Bacteroidetes are mostly affected by prenatal Valproate exposure [42]. Generally, these studies explained the correlation of elevated levels of Lactobacillus and Clostridium with the underlying inflammation. Unlike the diverse Lactobacillus genus, our results focus on the Lactobacillus ruminis species which is a known Tumor Necrosis Factor (TNF)-stimulatory strain [43].

Brain imaging analyses ( $\left.{ }^{1} \mathrm{H}-\mathrm{MRS}\right)$ in UHR subjects have already been performed for several years. Early in 2003, Wood et. al. reported a significant elevation of Choline/Creatine in the left dorsolateral prefrontal cortex (DLPFC) of UHR [44]. Other than the prefrontal cortex, the limbic system is increasingly being regarded as having an important role in the early damaging effects of schizophrenia [45]. Several studies have already reported schizophrenia patients and high-risk individuals have increased choline level in ACC [46-48], which is a significant part of the limbic system and a central hub for cognitive processing [49]. All mentioned observations are not only consistent with our findings but also leading to the interpretation that elevated choline level is a sign of disturbed membrane metabolism, often the result of glial activation [50].

\subsection{Gut microbiome and membrane hypothesis}

The membrane hypothesis has been greatly enriched and developed in recent years due to the joining of the microglia hypothesis [51] and the immune hypothesis [52]. Both emerging hypotheses emphasize the significant role of microglial activation in membrane dysfunction, which could induce excessive synaptic pruning, apoptosis, blood-brain barrier (BBB) disruption and amplification of neuroinflammation and then cause damage to the membrane integrity [53-56]. The evidences of activated microglia are heavily supported in the schizophrenia research field. For example, postmortem brain studies of schizophrenia have shown that the marker for activated microglia is elevated in patients [57,58]; a positron emission tomography (PET) brain imaging study also has suggested increased microglial activity in schizophrenia patients and UHR individuals [59]. However, the pathogenic mechanism of microglia activation in schizophrenia patients remains unclear. Genetically, the complement component 4 (C4) genes may stimulate the microglia via the complement cascade [60]. But how about the environmental factors, especially gut microbiome? To answer this, our results provided a possibility that changes in the gut microbiota may serve as a resource in microglial activation which contributes to the onset of schizophrenia based on membrane hypothesis.

Our main finding is that levels of Clostridiales, Prevotella and Lactobacillus ruminis in gut microbiota and level of choline in ACC are elevated in UHR subjects. As mentioned above, the association between elevated choline and membrane hypothesis is widely recognized, hence the role of gut microbiome in membrane hypothesis is the key point at present. In regard to the association between gut microbiota and the membrane hypothesis, the former two genera (Clostridiales and Prevotella) are known to be involved in the fermentation of carbohydrates and are prominent producers of SCFAs [61] which is also supported by our functional prediction. The pyruvate synthesis, acetyl-CoA synthesis and fatty acid biosynthesis initiation are three fundamental reactions in the synthesis of SCFAs [62] which were observed to be more active in UHR subjects to different degrees in our study. SCFAs, which mainly consist of acetate, propionate and butyrate, are able to cross the blood brain barrier (BBB) and may have different effects on microglia [63]. Studies have reported how microglial activation is affected by propionate and the microglial inhibition by butyrate $[64,65]$. However, high concentration and mixtures of SCFAs were suggested to promote activation of microglia and amplification of the inflammatory process $[66,67]$. In autism studies, total SCFAs were observed at increased levels in the stool of autistic children [68], and propionate was proven to induce autistic behaviors and microglia activation in animal models $[69,70]$. The administration of valproic acid, an effective assistance drug in alleviating schizophrenic symptoms, could inhibit the transport of SCFAs across the BBB in rodents [71], that in turn indicates the negative effect of SCFAs on brain function. As for our latter finding, taxon Lactobacillus ruminis could stimulate the production of the TNF [43]. There are three facts about TNF that most attract our interest: TNF could not only cross the BBB, but also increase the permeability of the BBB [72,73]; it is a robust activator of microglia [74] and the expression of TNF could be promoted by SCFAs [75]. So far, we could infer that the gut microbiota differences in UHR subjects may be involved in microglial-induced membrane dysfunction through elevating the SCFAs and TNF level, and thereby become the gut extension of schizophrenia's membrane hypothesis (Fig. 5). In addition, there is conceivable evidence suggesting that exposure to certain microbial proteins and inflammatory metabolites could weaken the BBB [76,77]. The membrane hypothesis could in turn be the brain extension to microbial translocation mechanism.

As for the HR and HC groups, our analyses did not show apparent distinction between this two groups. The HRs' relatively normal microbial environment may protect them from illness thereby counteracting risks brought on by their genes.

\subsection{Limitations and highlights}

The main limitation of this study is the small sample size, especially of the UHR group, which might make the direct relationship of choline concentration in brain and gut microbiota composition underpowered. Second, neither the metabolites (such as SCFAs and choline) nor the inflammation markers (including TNF) concentration in blood, stool or cerebrospinal fluid samples had been evaluated directly. Third, the Body Mass Index (BMI) and diet information are lacking in our study, though the dietary habits of individuals in Hunan Province are very similar. As the BMI and diet could have a nonnegligible impact on gut microbiota, we should control for these factors to decrease possible bias in our future study. Fourth, the phylogenetic power of 16S rRNA gene sequencing data is low at the species level [78]. Although the V4 region of the 16S rRNA gene is perceived to have relatively robust discrimination among most bacterial species [79], our species level result need to be treated carefully and replicated by future metagenomics sequencing. Fifth, our functional profiling is performed on the basis of 16S rRNA gene data [28]. We make inferences about the genome corresponding to the specific marker gene sequence, as well as about the functional potential of a given genome. Our current knowledge of metabolic pathways and functions is limited and we can estimate that the coverage is at 50\% of any given genome at best $[80,81]$. Most of the well-characterized functions correspond to housekeeping genes. It is likely that more relevant pathways will be discovered in the future, as we develop better high-throughput methods to either characterize or predict the functions of microbial genes which would match the pace of our discoveries [82]. Finally, our ${ }^{1} \mathrm{H}$ MRS scans only include one region of interest (ROI) - the anterior cingulate cortex (ACC), prompting the need for more areas of the brain to be observed.

Notably, we conducted the gut microbiome and ${ }^{1} \mathrm{H}$ MRS study in the same subjects at the same time thus strengthening our conclusions. All the subjects we studied were drug-naïve for at 


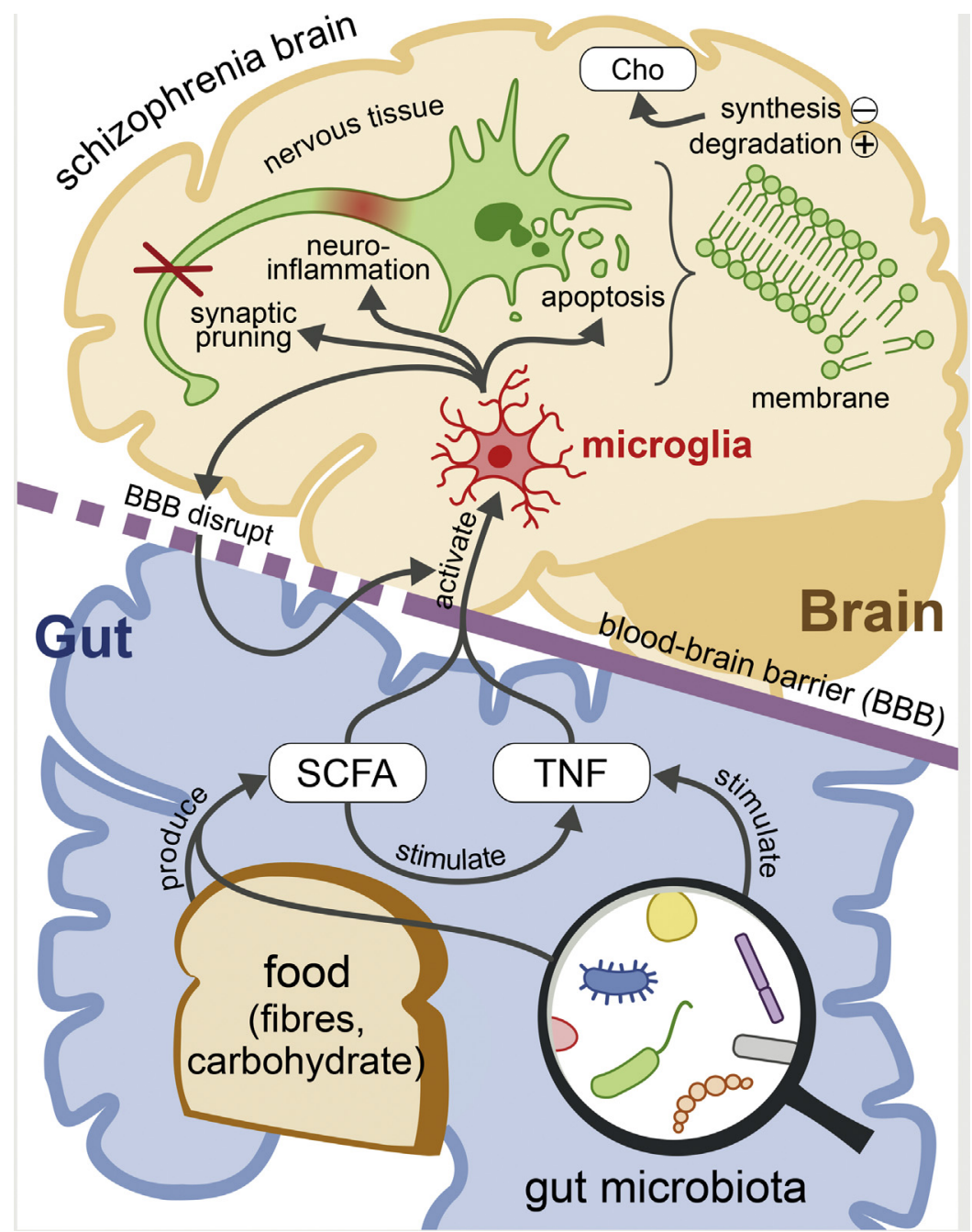

Fig. 5. Schematic representation of the gut microbiota promotes the onset of schizophrenia via the membrane hypothesis.

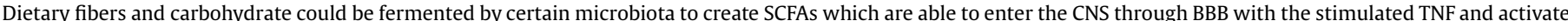

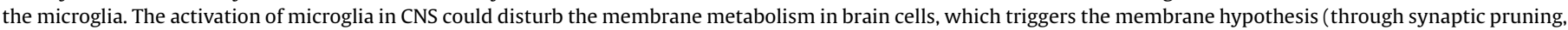

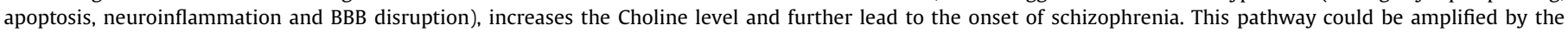
increased permeability of BBB.

Abbreviations: SCFAs: Short Chain Fatty Acids; TNF: Tumor Necrosis Factor; CNS: Central Nervous System; BBB: Blood-brain barrier; Cho: choline.

least 3 months, hence excluding one important confounding effect of gut microbiome. Our exploration bridges the gap between brain membrane metabolism and gut microbiota with the potential microglial activation by SCFAs. This baseline study of UHR subjects provides a new perspective for the pathological process of schizophrenia. In a follow-up study, we will test the hypotheses formulated in this paper by collecting longitudinal microbiome and brain imaging data to record the trajectory of transition to psychosis in high-risk subjects. Consequently, we aim to build a predictive microbiome-brain model of schizophrenia.

Our findings strengthen the link between the gut microbiome and schizophrenia via the membrane hypothesis. It gives hope for the use of the gut microbiota as a tool for early diagnosis of schizophrenia as well as the development of personalized microbial therapeutics, thereby alleviating psychiatric symptoms in a precision-medicine approach.

\section{Author contributions}

YH, JT and XC designed the study. YH, YZ, ZL, XM, NY and LY collected the samples and clinical informations. $\mathrm{CL}$ and $\mathrm{KJ}$ carried out the brain scaning. YH, TK, QZ, MT and RK analyzed and discussed the experimental result. $\mathrm{YH}$ and TK wrote the first draft of the manuscript. All authors contributed to and have approved the final manuscript.

\section{Conflict of interest}

There is no conflict of interest to declare.

\section{Acknowledgements}

This work was funded by the National Natural Science Foundation of China (81471361 and 81271484 to X.C.) and China Scholarship Council (Grant No. 201606370199 to Y.H.). We thank all the participants who donated their stool samples and cooperated with the brain scanning and evaluation. And we would like to thank Dr. Stephen J. Glatt and Mr. Jason Zhao for constructive comments and revisions.

\section{Appendix A. Supplementary data}

Supplementary data associated with this article can be found, in the online version, at https://doi.org/10.1016/j.eurpsy.2018.05.011. 


\section{References}

[1] McGrath J., Saha S, Chant D, Welham J. Schizophrenia A concise overview of incidence, prevalence, and mortality. Epidemiol Rev 2008;30:67-76, doi: http://dx.doi.org/10.1093/epirev/mxn001.

[2] Cardno AG, Marshall EJ, Coid B, Macdonald AM, Ribchester TR, Davies NJ, et al. Heritability estimates for psychotic disorders: the Maudsley Twin psychosis series. Arch Gen Psychiatry 1999;56:162-8, doi:http://dx.doi.org/10.1001/ archpsyc.56.2.162.

[3] Clemente CJ, Ursell KL, Parfrey WL, Knight R. The impact of the gut microbiota on human health: an integrative view. Cell 2012;148:1258-70.

[4] Li J, Jia H, Cai X, Zhong H, Feng Q, Sunagawa S, et al. An integrated catalog of reference genes in the human gut microbiome. Nat Biotechnol 2014;32:83441, doi:http://dx.doi.org/10.1038/nbt.2942.

[5] Lloyd-Price J, Mahurkar A, Rahnavard G, Crabtree J, Orvis J, Hall AB, et al. Strains, functions and dynamics in the expanded Human Microbiome Project. Nature 2017;550:61-6, doi:http://dx.doi.org/10.1038/nature23889.

[6] Qin J, Li R, Raes J, Arumugam M, Burgdorf SK, Manichanh C, et al. A human gut microbial gene catalogue established by metagenomic sequencing. Nature 2010;464:59-65.

[7] Sender R, Fuchs S, Milo R. Revised estimate for the number of human and bacterial cells in the body. BioRvix 2016;14:1-21, doi:http://dx.doi.org/ $10.1101 / 036103$.

[8] Rhee SH, Pothoulakis C, Mayer EA. Principles and clinical implications of the brain-gut-enteric microbiota axis. Nat Rev Gastroenterol Hepatol 2009;6:30614, doi:http://dx.doi.org/10.1038/nrgastro.2009.35.

[9] Dinan TG, Cryan JF. The impact of gut microbiota on brain and behaviour: implications for psychiatry. Curr Opin Clin Nutr Metab Care 2015;18:552-8, doi:http://dx.doi.org/10.1097/MCO.0000000000000221.

[10] Smith AP. The tantalizing links between gut microbes and the brain. Nature 2015;526:312-4.

[11] Carlsson A, Lindqvist M. Effect of chlorpromazine or haloperidol on formation of 3methoxytyramine and normetanephrine in mouse brain. Acta Pharmacol Toxicol (Copenh) 2016;196020:140.

[12] Woolley DW, Weil-Malherbe H. The biochemical bases of psychoses. J Nerv Ment Dis 1964;138:92, doi:http://dx.doi.org/10.1097/00005053-196401000-00014.

[13] Kim JS, Kornhuber HH, Schmid-Burgk W, Holzmüller B. Low cerebrospinal fluid glutamate in schizophrenic patients and a new hypothesis on schizophrenia. Neurosci Lett 1980;20:379-82, doi:http://dx.doi.org/10.1016/ 0304-3940(80)90178-0.

[14] Horrobin DF, Glen AIM, Vaddadi K. The membrane hypothesis of schizophrenia. Schizophr Res 1994;13:195-207, doi:http://dx.doi.org/ 10.1016/0920-9964(94)90043-4.

[15] Dinan TG, Stilling RM, Stanton C, Cryan JF. Collective unconscious: how gut microbes shape human behavior. J Psychiatr Res 2015;6:3, doi:http://dx.doi. org/10.1016/j.jpsychires.2015.02.021.

[16] Gershon MD, Tack J. The serotonin signaling system: from basic understanding to drug development for functional GI disorders. Gastroenterology 2007;132:397-414, doi:http://dx.doi.org/10.1053/j.gastro.2006.11.002.

[17] Yano JM, Yu K, Donaldson GP, Shastri GG, Ann P, Ma L, et al. Indigenous bacteria from the gut microbiota regulate host serotonin biosynthesis. Cell 2015;161:264-76, doi:http://dx.doi.org/10.1016/j.cell.2015.02.047.

[18] Kemp van der WJM. Explorations into the membrane hypothesis of schizophrenia. 2011.

[19] Shutter L, Tong KA, Holshouser BA. Proton MRS in acute traumatic brain injury: role for glutamate/glutamine and choline for outcome prediction. J Neurotrauma 2004;21:1693-705, doi:http://dx.doi.org/10.1089/ neu.2004.21.1693.

[20] Shen Y, Xu J, Li Z, Huang Y, Yuan Y, Wang J, et al. Analysis of gut microbiota diversity and auxiliary diagnosis as a biomarker in patients with schizophrenia: a cross-sectional study. Schizophr Res 2018, doi:http://dx.doi. org/10.1016/j.schres.2018.01.002.

[21] Schwarz E, Maukonen J, Hyytiäinen T, Kieseppä T, Orešič M, Sabunciyan S, et al. Analysis of microbiota in first episode psychosis identifies preliminary associations with symptom severity and treatment response. Schizophr Res 2017, doi:http://dx.doi.org/10.1016/j.schres.2017.04.017.

[22] APA. Diagnostic and statistical manual of mental disorders. Am Psychiatr Assoc 2000.

[23] McGlashan HT, Miller JT, Woods WS, Rosen LJ, Hoffman ER, Davidson L. Structured interview for prodromal syndromes. PRIME Res Clin Yale Sch Med N Hav CT 1997.

[24] Hall R. Global assessment of functioning: a modified scale. Psychosomatics 1995;3:6, doi:http://dx.doi.org/10.1016/S0033-3182(95)71666-8.

[25] Callahan JB, McMurdie JP, Rosen JM, Han WA, Johnson AAJ, Holmes PS. DADA2. High-resolution sample inference from Illumina amplicon data. Nat Methods 2016;13:581-3.

[26] Caporaso JG, Kuczynski J, Stombaugh J, Bittinger K, Bushman FD, Costello EK, et al. QIIME allows analysis of high-throughput community sequencing data. Nat Methods 2010;7:335-6, doi:http://dx.doi.org/10.1038/nmeth.f.303.

[27] Segata N, Izard J, Waldron L, Gevers D, Miropolsky L, Garrett WS, et al Metagenomic biomarker discovery and explanation. Genome Biol 2011;1:2, doi:http://dx.doi.org/10.1186/gb-2011-12-6-r60.

[28] Langille IMG, Zaneveld J, Caporaso GJ, McDonald D, Knights D, Reyes AJ, et al. Predictive functional profiling of microbial communities using 16S rRNA marker gene sequences. Nat Biotechnol 2013;31:814-21.
[29] Parks DH, Tyson GW, Hugenholtz P. Beiko RG. STAMP. Statistical analysis of taxonomic and functional profiles. Bioinformatics 2014;30:3123-4, doi:http:// dx.doi.org/10.1093/bioinformatics/btu494.

[30] Lance NG, Williams TW. Mixed-Data classificatory programs I - agglomerative systems. Aust Comput J 1963;1.

[31] Stilling RM, van de Wouw M, Clarke G, Stanton C, Dinan TG, Cryan JF. The neuropharmacology of butyrate: the bread and butter of the microbiota-gutbrain axis? Neurochem Int 2016;99:110-32, doi:http://dx.doi.org/10.1016/j. neuint.2016.06.011.

[32] Macfarlane S, Macfarlane GT. Regulation of short-chain fatty acid production. Proc Nutr Soc 2003;62:67-72, doi:http://dx.doi.org/10.1079/PNS2002207.

[33] Adams JB, Johansen LJ, Powell LD, Quig D, Rubin RA. Gastrointestinal flora and gastrointestinal status in children with autism - comparisons to typical children and correlation with autism severity. BMC Gastroenterol 2011;11:22, doi:http://dx.doi.org/10.1186/1471-230X-11-22.

[34] Strati F, Cavalieri D, Albanese D, De Felice C, Donati C, Hayek J, et al. New evidences on the altered gut microbiota in autism spectrum disorders. Microbiome 2017;5:24, doi:http://dx.doi.org/10.1186/s40168-017-0242-1.

[35] Tomova A, Husarova V, Lakatosova S, Bakos J, Vlkova B, Babinska K, et al. Gastrointestinal microbiota in children with autism in Slovakia. Physiol Behav 2015;138:179-87.

[36] Angelis M, Piccolo M, Vannini L, Siragusa S, Giacomo A, Serrazzanetti D, et al. Fecal microbiota and metabolome of children with autism and pervasive developmental disorder not otherwise specified. PLoS One 2013;8, doi:http:/ dx.doi.org/10.1371/journal.pone.0076993.

[37] Finegold SM, Molitoris D, Song Y, Liu C, Vaisanen M, Bolte E, et al. Gastrointestinal microflora studies in late-onset autism. Clin Infect Dis 2002;35:S6-16, doi:http://dx.doi.org/10.1086/341914.

[38] Parracho HMRT, Bingham MO, Gibson GR, McCartney AL. Differences between the gut microflora of children with autistic spectrum disorders and that of healthy children. J Med Microbiol 2005;54:987-91, doi:http://dx.doi.org/ $10.1099 / \mathrm{jmm} \cdot 0.46101-0$

[39] Song Y, Liu C, Finegold SM. Real-time PCR quantitation of clostridia in feces of autistic children. Appl Environ Microbiol 2004;70:6459-65, doi:http://dx.doi. org/10.1128/AEM.70.11.6459-6465.2004.

[40] Castro-Nallar E, Bendall ML, Pérez-Losada M. Composition, taxonomy and functional diversity of the oropharynx microbiome in individuals with schizophrenia and controls. PeerJ 2015.

[41] Yolken RH, Severance EG, Sabunciyan S, Gressitt KL, Chen O, Stallings C, et al Metagenomic sequencing indicates that the oropharyngeal phageome of individuals with schizophrenia differs from that of controls. Schizophr Bull 2015;41:1153-61, doi:http://dx.doi.org/10.1093/schbul/sbu197.

[42] de Theije CGM, Wopereis H, Ramadan M, van Eijndthoven T, Lambert J, Knol J, et al. Altered gut microbiota and activity in a murine model of autism spectrum disorders. Brain Behav Immun 2014;37:197-206, doi:http://dx.doi. org/10.1016/j.bbi.2013.12.005

[43] Taweechotipatr M, Iyer C, Spinler KJ, Versalovic J, Tumwasorn S. Lactobacillus saerimneri and Lactobacillus ruminis: novel human-derived probiotic strains with immunomodulatory activities. FEMS Microbiol Lett 2009;293:65-72.

[44] Wood JS, Berger G, Velakoulis D, Phillips JL, McGorry DP, Yung RA, et al. Proton magnetic resonance spectroscopy in first episode psychosis and ultra high-risk individuals. Schizophr Bull 2001;29:831.

[45] Copolov D, Velakoulis D, Mcgorry P, Mallard C, Yung A, Rees S, et al. Neurobiological findings in early phase schizophrenia 1. Brain Res Rev 2000;31:157-65.

[46] O’Neill J, Levitt J, Caplan R, Asarnow R, McCracken TJ, Toga WA, et al. 1H MRS evidence of metabolic abnormalities in childhood-onset schizophrenia. Neuroimage 2004;21:1781-9.

[47] Tandon N, Bolo NR, Sanghavi K, Mathew IT, Francis AN, Stanley JA, et al. Brain metabolite alterations in young adults at familial high risk for schizophrenia using proton magnetic resonance spectroscopy. Schizophr Res 2013;148:5966, doi:http://dx.doi.org/10.1016/j.schres.2013.05.024.

[48] Chiu PW, Lui SSY, Hung KSY, Chan RCK, Chan Q, Sham PC, et al. In vivo gammaaminobutyric acid and glutamate levels in people with first-episode schizophrenia: a proton magnetic resonance spectroscopy study. Schizophr Res 2017, doi:http://dx.doi.org/10.1016/j.schres.2017.07.021.

[49] Cordes JS, Mathiak KA, Dyck M, Alawi EM, Gaber TJ, Zepf FD, et al. Cognitive and neural strategies during control of the anterior cingulate cortex by fMRI neurofeedback in patients with schizophrenia. Front Behav Neurosci 2015;9, doi:http://dx.doi.org/10.3389/fnbeh.2015.00169.

[50] Plitman E, de la Fuente-Sandoval C, Reyes-Madrigal F, Chavez S, Gómez-Cruz C, León-Ortiz P, et al. Elevated myo-inositol, choline, and glutamate levels in the associative striatum of antipsychotic-naive patients with first-episode psychosis: a proton magnetic resonance spectroscopy study with implications for glial dysfunction. Schizophr Bull 2016;42:415-24, doi:http://dx.doi.org/ $10.1093 /$ schbul/sbv118.

[51] Monji A, Kato T, Kanba S. Cytokines and schizophrenia. Microglia hypothesis of schizophrenia. Psychiatry Clin Neurosci 2009;63:257-65.

[52] Kinney KD, Hintz K, Shearer ME, Barch HD, Riffin C, Whitley K, et al. A unifying hypothesis of schizophrenia: abnormal immune system development may help explain roles of prenatal hazards, post-pubertal onset, stress, genes, climate, infections, and brain dysfunction. Med Hypotheses 2010;74:555-63.

[53] da Fonseca ACC, Matias D, Garcia C, Amaral R, Geraldo LH, Freitas C, et al. The impact of microglial activation on blood-brain barrier in brain diseases. Front Cell Neurosci 2014;8:362, doi:http://dx.doi.org/10.3389/fncel.2014.00362. 
[54] Minghetti L, Ajmone-Cat MA, De Berardinis MA, De Simone R. Microglial activation in chronic neurodegenerative diseases: roles of apoptotic neurons and chronic stimulation. Brain Res Rev 2005;48:251-6, doi:http://dx.doi.org/ 10.1016/j.brainresrev.2004.12.015.

[55] Paolicelli RC, Bolasco G, Pagani F, Maggi L, Scianni M, Panzanelli P, et al. Synaptic pruning by microglia is necessary for normal brain development. Science 2011;333:1456-8, doi:http://dx.doi.org/10.1126/science.1202529.

[56] Streit WJ, Mrak RE, Griffin WST. Microglia and neuroinflammation. A pathological perspective. J Neuroinflammation 2004;1, doi:http://dx.doi.org/ 10.1186/1742-2094-1-14.

[57] Bayer TA, Buslei R, Havas L, Falkai P. Evidence for activation of microglia in patients with psychiatric illnesses. Neurosci Lett 1999;271:126-8.

[58] Radewicz K, Garey LJ, Gentleman SM, Reynolds R. Increase in HLA-DR immunoreactive microglia in frontal and temporal cortex of chronic schizophrenics. J Neuropathol Exp Neurol 2000;59:137-50, doi:http://dx.doi. org/10.1093/jnen/59.2.137.

[59] Bloomfield SP, Selvaraj S, Veronese M, Rizzo G, Bertoldo A, Owen RD, et al. Microglial activity in people at ultra high risk of psychosis and in schizophrenia: an [(11)C]PBR28 PET brain imaging study. Am J Psychiatry 2016;173:44-52.

[60] Sekar A, Bialas AR, De Rivera H, Davis A, Hammond TR, Kamitaki N, et al Schizophrenia risk from complex variation of complement component 4. Nature 2016;530:177-83, doi:http://dx.doi.org/10.1038/nature16549.

[61] Houser MC, Tansey MG. The gut-brain axis: is intestinal inflammation a silent driver of Parkinson's disease pathogenesis? Npj Park Dis 2017;3, doi:http://dx. doi.org/10.1038/s41531-016-0002-0.

[62] Tumanov S, Bulusu V, Kamphorst JJ. Methods in enzymology. Methods Enzymol 2015;561:197-217, doi:http://dx.doi.org/10.1016/bs.mie.2015.05.017.

[63] Vadder F, Petia K-D, Goncalves D, Vinera J, Zitoun C, Duchampt A, et al. Microbiota-generated metabolites promote metabolic benefits via gut-brain neural circuits. Cell 2014;156:84-96, doi:http://dx.doi.org/10.1016/j. cell.2013.12.016.

[64] Sherwin E, Sandhu KV, Dinan TG, Cryan JF. May the force Be with you: the light and dark sides of the microbiota-gut-brain axis in neuropsychiatry. CNS Drugs 2016;30:1019-41, doi:http://dx.doi.org/10.1007/s40263-016-0370-3.

[65] Vinolo MAR, Rodrigues HG, Nachbar RT, Curi R. Regulation of inflammation by short chain fatty acids. Nutrients 2011;3:858-76, doi:http://dx.doi.org/ $10.3390 /$ nu3100858.

[66] Pender LS, Quinn JJ, Sanderson RI, MacDonald TT. Butyrate upregulates stromelysin-1 production by intestinal mesenchymal cells. Am J Physiol Gastrointest Liver Physiol 2000;279:G918-24.

[67] Sampson TR, Debelius JW, Thron T, Janssen S, Shastri GG, Ilhan ZE, et al. Gut microbiota regulate motor deficits and neuroinflammation in a model of parkinson's disease. Cell 2016;167:1469-80, doi:http://dx.doi.org/10.1016/j. cell.2016.11.018 e12.

[68] Wang L, Christophērsen CT, Sorich MJ, Gerber JP, Angley MT, Conlon MA Elevated fecal short chain fatty acid and ammonia concentrations in children with autism spectrum disorder. Dig Dis Sci 2012;57:2096-102, doi:http://dx. doi.org/10.1007/s10620-012-2167-7.

[69] Ossenkopp KP, Foley KA, Gibson J, Fudge MA, Kavaliers M, Cain DP, et al. Systemic treatment with the enteric bacterial fermentation product, propionic acid, produces both conditioned taste avoidance and conditioned place avoidance in rats. Behav Brain Res 2012;227:134-41, doi:http://dx.doi.org/ 10.1016/j.bbr.2011.10.045.

[70] Shultz SR, MacFabe DF Martin S, Jackson J, Taylor R, Boon F, et al. Intracerebroventricular injections of the enteric bacterial metabolic product propionic acid impair cognition and sensorimotor ability in the Long-Evans rat: further development of a rodent model of autism. Behav Brain Res 2009;200:33-41, doi:http://dx.doi.org/10.1016/j.bbr.2008.12.023.

[71] Adkison DK, Shen D. Uptake of vaiproic acid into rat brain is mediated fatty acid transporter. J Pharmacol Exp Ther 1996;276:1189-200.

[72] Banks WA, Kastin AJ, Broadwell RD. Passage of cytokines across the bloodBrain barrier. Neuroimmunomodulation 1996;2:241-8, doi:http://dx.doi.org/ $10.1159 / 000097202$.

[73] Tsao N, Hsu HPP, Liu CMM, Liu CCC, Lei HYY, Wu CMM, et al. Tumour necrosis factor-(causes an increase in blood-brain barrier permeability during sepsis. J Med Microbiol 2001;50:812-21, doi:http://dx.doi.org/10.1099/0022-1317-509-812.

[74] Klapal L, Igelhorst BA, Dietzel-Meyer ID. Changes in neuronal excitability by activated microglia: differential $\mathrm{Na}+$ current upregulation in pyramid-shaped and bipolar neurons by TNF- $\alpha$ and IL-18. Front Neurol 2016;7, doi:http://dx.doi. org/10.3389/fneur.2016.00044.

[75] Morris G, Berk M, Carvalho A, Caso JR, Sanz Y, Walder K, et al. The role of the microbial metabolites including tryptophan catabolites and short chain fatty acids in the pathophysiology of immune-Inflammatory and neuroimmune disease. Mol Neurobiol 2017;54:4432-51, doi:http://dx.doi.org/10.1007/ s12035-016-0004-2.

[76] Downs R, Perna J, Vitelli A, Cook D, Dhurjati P. Model-based hypothesis of gut microbe populations and gut/brain barrier permeabilities in the development of regressive autism. Med Hypotheses 2014;83:649-55, doi:http://dx.doi.org/ 10.1016/j.mehy.2014.09.005.

[77] Huang S-H, Jong AY. Cellular mechanisms of microbial proteins contributing to invasion of the blood-brain barrier. \{MicroReview\}. Cell Microbiol 2001;3:277-87, doi:http://dx.doi.org/10.1046/j.1462-5822.2001.00116.x.

[78] Janda JM, Abbott SL. 16S rRNA gene sequencing for bacterial identification in the diagnostic laboratory: pluses, perils, and pitfalls. J Clin Microbiol 2007;45:2761-4, doi:http://dx.doi.org/10.1128/JCM.01228-07.

[79] Caporaso JG, Lauber CL, Walters WA, Berg-Lyons D, Lozupone CA, Turnbaugh PJ, et al. Global patterns of $16 \mathrm{~S}$ rRNA diversity at a depth of millions of sequences per sample. Proc Natl Acad Sci 2011;108:4516-22, doi:http://dx.doi. org/10.1073/pnas.1000080107.

[80] Mi H, Muruganujan A, Thomas PD. PANTHER in 2013: Modeling the evolution of gene function, and other gene attributes, in the context of phylogenetic trees. Nucleic Acids Res 2013;4:1, doi:http://dx.doi.org/10.1093/nar/gks1118.

[81] Suzek BE, Wang Y, Huang H, McGarvey PB, Wu CH. UniRef clusters: a comprehensive and scalable alternative for improving sequence similarity searches. Bioinformatics 2015;31:926-32, doi:http://dx.doi.org/10.1093/ bioinformatics/btu739.

[82] Jiang Y, Oron TR, Clark WT, Bankapur AR, D'Andrea D, Lepore R, et al. An expanded evaluation of protein function prediction methods shows an improvement in accuracy. Genome Biol 2016;17:184, doi:http://dx.doi.org/ $\underline{10.1186 / s 13059-016-1037-6 .}$ 